\title{
Optimization of Collaborative Spectrum Sensing Mechanism for Cognitive Radio
}

\author{
Zhigang Wen ${ }^{*}$ and Mingjun $\mathrm{Mu}$
}

School of Electronic Engineering Beijing, University of Posts and Telecommunications, 100876, Beijing, P.R. China

\begin{abstract}
Cognitive radio (CR) is an emerging technology that helps efficiently use spectrum. In this paper, a collaborative spectrum sensing mechanism is proposed which combines passive detection and sensing service compensation. Theoretical analysis and simulations show that this method not only reduces the energy cost and the passive sensing time, but also maintains fairness in the CR communication systems.
\end{abstract}

Keywords: Cognitive radio, Collaborative spectrum sensing, Detection time, Sensing mechanism.

\section{INTRODUCTION}

With the development of wireless communication technology, the contradiction between performance and cost has been increasing. The development is blocked by the scarcity of spectrum resources and the inefficiency of static spectrum allocation strategy. To improve spectrum utilization, Dr. Jeseph Mitola proposed "cognitive radio" [1]. Its core idea is that the wireless communication device can find the "spectrum hole" in the licensed spectrum band, and take advantage of it with no interference to the primary user (PU). This technology can significantly improve the utilization of spectrum resources and effectively alleviate the problem of spectrum scarcity.

Before the secondary user (SU) can send data on the licensed spectrum, it has to percept the spectrum accurately; therefore, spectrum sensing is one of the key technologies in cognitive radio. At present, the spectrum detect precision of single-node is affected by the multipath fading and shadow because of the location of node; besides the computational capability of single-node is limited. As a result, its detection performance is not good. The cooperative spectrum sensing technology can overcome the above shortcomings, but there are a lot of contradictions between performance and efficiency. How to improve the efficiency of spectrum sensing and spectrum utilization is the key to collaborative spectrum sensing. Several researches and optimizations for cooperation spectrum sensing have been reported [2-9]. Distributed collaboration network structure is researched [24] without considering cognitive users' computing burden, and perception time and throughput is optimized [5]. How to lighten the cognitive networks burden is researched $[6,7]$ in which the author maximizes the spectrum sensing efficiency in Gaussian noise channel and Rayleigh fading channel

*Address correspondence to this author at the School of Electronic Engineering Beijing, University of Posts and Telecommunications, 100876, Beijing, P.R. China; Tel: 0086-10-62283467; Fax: 0086-10-62283467;

E-mail: zwen@bupt.edu.cn
[8]. All of these studies have adopted active spectrum sensing mode without considering the energy cost of cognitive users. As highlighted in reference [9], active spectrum sensing is set as a cycle, but it is unrealistic to force all users to periodically percept to maintain the whole networks. In response to these problems, a collaborative passive spectrum sensing mode combined with sensing service compensation is proposed. Passive spectrum sensing mode can reduce the average energy cost of the whole cognitive networks, and the sensing service compensation can shorten the spectrum sensing time to make up for the shortcoming of passive mode.

The rest of the paper is organized as follows: section 2 introduces the proposed model which combines passive spectrum sensing mode with sensing service compensation; section 3 analyzes the feasibility of proposed model and addresses the optimization for the performance of spectrum sensing from simulation results; section 4 describes the advantages of proposed model in energy cost; finally, section 5 draws conclusions.

\section{SYSTEM MODEL AND PROBLEM STATEMENT}

The passive spectrum sensing mode is known for its long perception time. And the shortcoming leads to missing the spectrum opportunities and making the channel switching delay longer. Before trying to find a new mechanism to overcome the shortcoming, the working mechanism of the traditional passive spectrum sensing is to be looked upon. Fig. (1) illustrates an SU's complete communication process. There are four time periods between "demand" and "start". If some periods can be removed before "start transmission", the efficiency of spectrum detection is improved. Based on the above ideas, a new model of passive spectrum is proposed in Fig. (2).

There is less difference between the two processes in total time. In the proposed program, periods of sensing and data integration are removed before transporting and spectrum sensing is carried out after the SU completes its transmission and data integration runs asynchronously along 
with the entire process. In the cooperative spectrum sensing scheme, one SU can take advantage of others' sensing result for its own service as long as results of other SUs are reliable enough. If one SU uses others' spectrum sensing result ignoring its own result, there is no need for the FC to fuse data synchronously. In this way, the period before data transmission is to be shortened for better performance of spectrum detection.

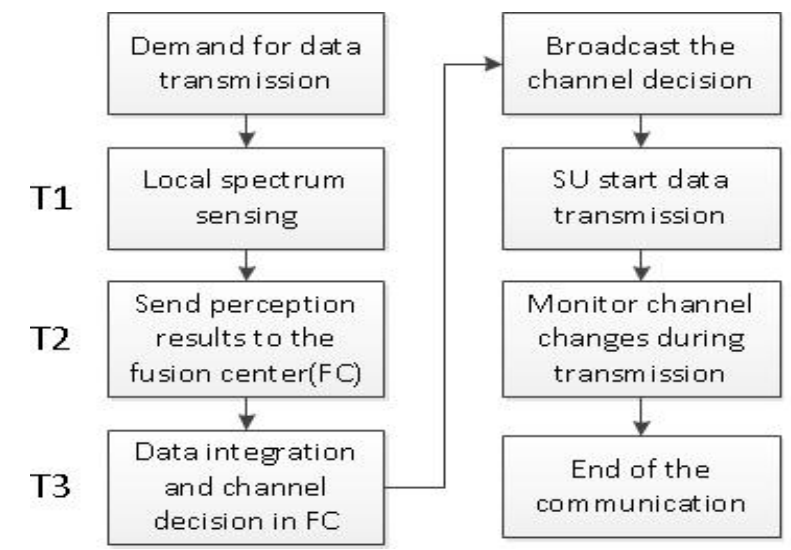

Fig. (1). The flow chart of traditional model.

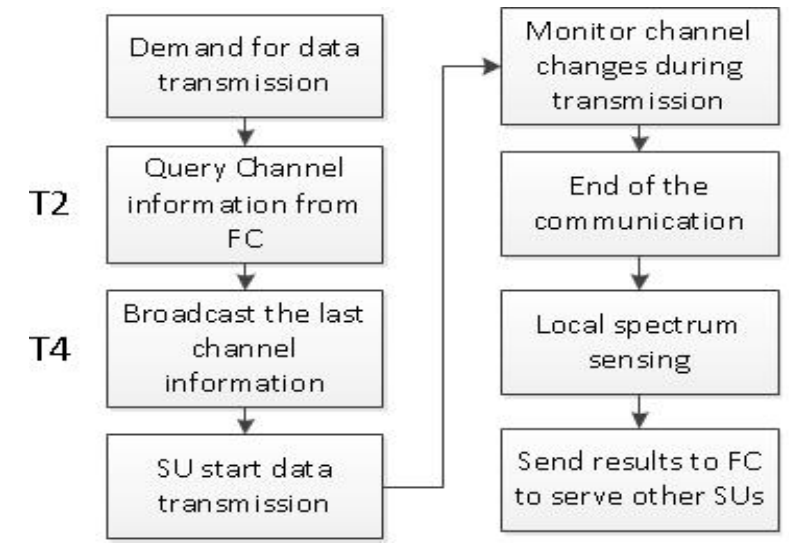

Fig. (2). The flow chart of new model.

The theoretical challenge faced by the proposed program is that SU must obey the principle of "listen before transmitting" through traditional spectrum sensing theory [10]. It is necessary for the single cognitive user because it is the only way through which it can get an available channel. However, in practical situation, single user affected by multipath fading and shadow performances gives unfavorable detection results. Cooperative spectrum sensing technology has been applied to solve this problem. In collaborative networks, several SUs send their local sensing results to FC where data is fused based on appropriate data fusion rules(such as "AND" "OR" "K/N", etc.). The final judgment is given by FC, and this result is improved compared to that of single users.

Assuming that there are $\mathrm{N}$ cognitive users which are independent and identically distributed in the network, the average SNR of each user is the same. Therefore, the probability of detection $(\mathrm{Pd})$ for each user is the same when energy detection method is carried out. No matter what kind of data integration is used, the quality of detection (Qd) in FC meets the detection precision (R) when $\mathrm{N}$ is large enough. With the "OR" fusion rule, for example, $\mathrm{N}$ is required to meet

$Q_{d}=1-\left(1-P_{d}\right)^{N} \geq R$

Simulation results in Fig. (3) show that with different Pd, $\mathrm{Qd}$ requires different $\mathrm{N}$ to reach detection precision (R). As $\mathrm{N}$ increases, Qd can always meet the requirements. Meanwhile, Qd almost increases no longer after $\mathrm{N}$ reaches to a certain point which can be set when Qd reaches 0.95 . Then the point is the optimal number (Nop) for coordination users, larger waste energy and smaller? leading to bad performance.

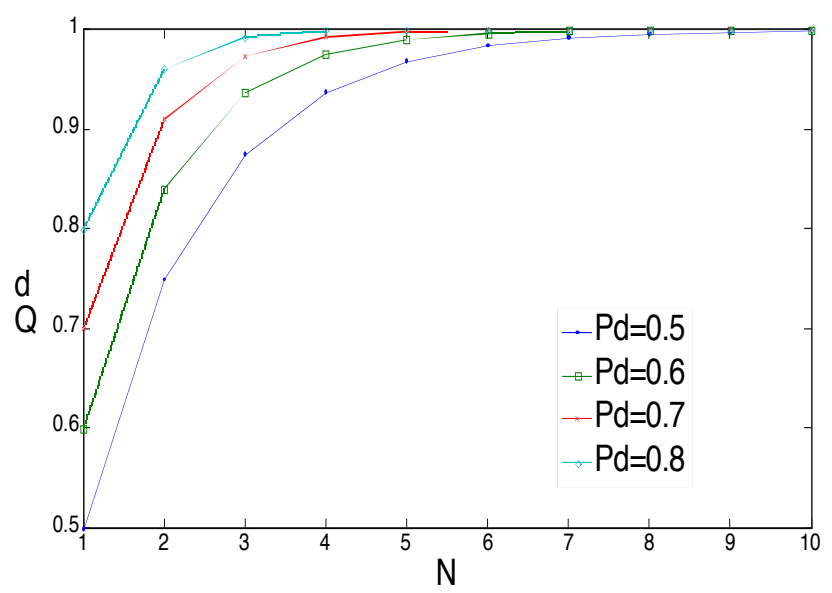

Fig. (3). the relationship between N, Pd and Qd

In the collaborative cognitive radio networks, there are several SUs to sense spectrum including the SU itself which has data transmission demand. If the number of these SUs is larger than Nop+1, the SU which needs transmission can skip its sensing period and use others' sensing result, so that, the sensing delay is shortened and the perception precision is guaranteed.

\section{PERFORMANCE SIMULATION}

The performance of the proposed scheme is simulated in MATLAB. A collaborative cognitive radio network is considered with 10 licensed channels changing randomly and uniformly and spectrum detection based on energy and the number of SUs in line with "Poisson Process". A survey of the average number of success detection was carried out in a period of time between traditional passive spectrum sensing model and proposed model under AWGN channel and Rayleigh channel. Fig. (4) shows that the proposed scheme improved the detection performance than the traditional passive detection scheme whether in AWGN channel or Rayleigh channel. And with the improvement of the SNR, the improved performance of proposed scheme appeared more pronounced and stabilized. As a result of the cooperative sensing strategy, the detecting precision in Rayleigh channel is close to that in AWGN channel. In certain situation, the detection performance of the proposed scheme under Rayleigh channel is better than that of the traditional scheme under AWGN channel. 


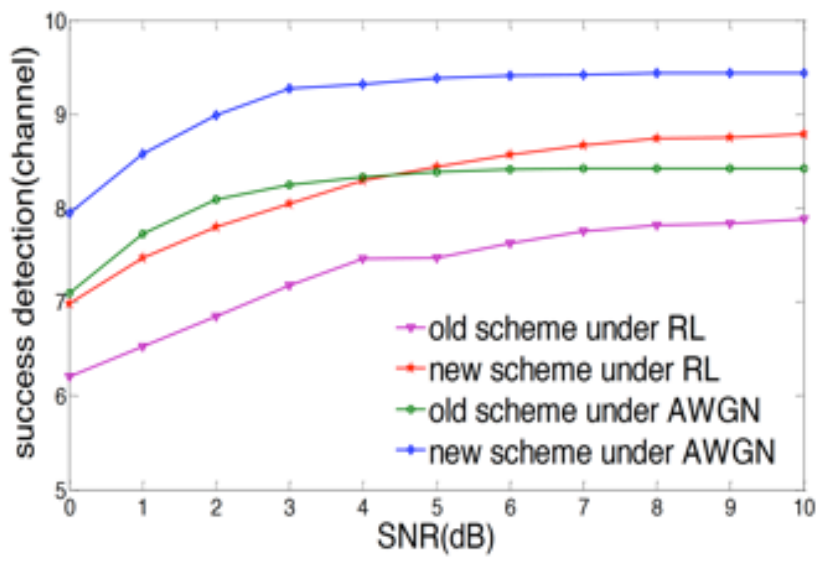

Fig. (4). performance of detection.

The change rate of licensed channel is another important factor to affect the detection performance of the two schemes. Fig. (5) shows that how much the proposed scheme can improve in detection number than the traditional scheme both in AWGN channel and in Rayleigh channel. X-axis represents channel numbers that the proposed scheme detects more than the traditional scheme, and Y-axis represents how often licensed channels change. Obviously, the faster detection scheme has more obvious advantage than the slower one in the case of fast changing of the channel. So the proposed scheme is more applicable in the networks where channel states change fast.

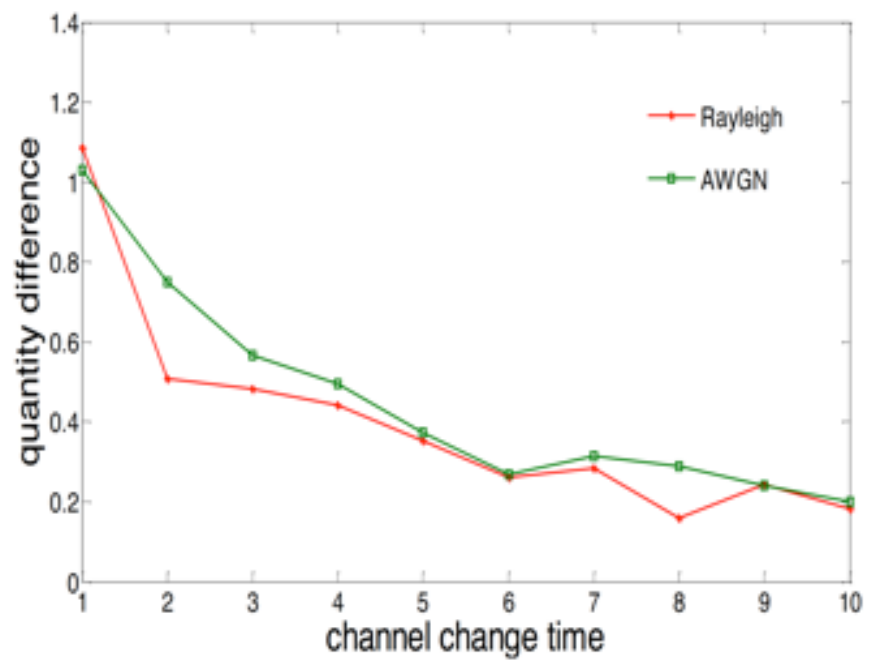

Fig. (5). Detection difference with channel change.

\section{OPTIMIZATION OF ENERGY CONSUMPTION}

Saving energy is another prominent advantage of the proposed scheme. As shown in Fig. (6), there are different SUs divided into active SU and sleep SU in the networks. An $\mathrm{SU}$ is active if it has data transmission demand, and goes through the process shown in Fig. (2), otherwise it sleeps. Compared with traditional scheme which requires all SUs to sense spectrum, the proposed scheme will obviously save a lot of energy.

A specific example is given to illustrate the proposed scheme on energy saving. Assuming that in a multimode and heterogeneous cognitive radio network, a large number of nodes are allowed to communicate at the same time. If there are some SUs (the number is equal or larger than Nop) completing their communication and spectrum sensing in a short period, FC can obtain the last information about the spectrum in this network. Any other SUs that have communication demand at this moment can ask the FC for channel state directly, and do not need to wait for the results of all SUs in this network. If the Pd of single $\mathrm{SU}$ is 0.7 , the FC just needs 4 SUs to reach high precision $(R>0.95)$. According to the U.S. Federal Communications Commission (FCC), the utilization of the licensed spectrum band is only $15 \%$ to $85 \%$ [11]. In the case when the idle spectrum is taken full advantage of, the above condition is easily satisfied.

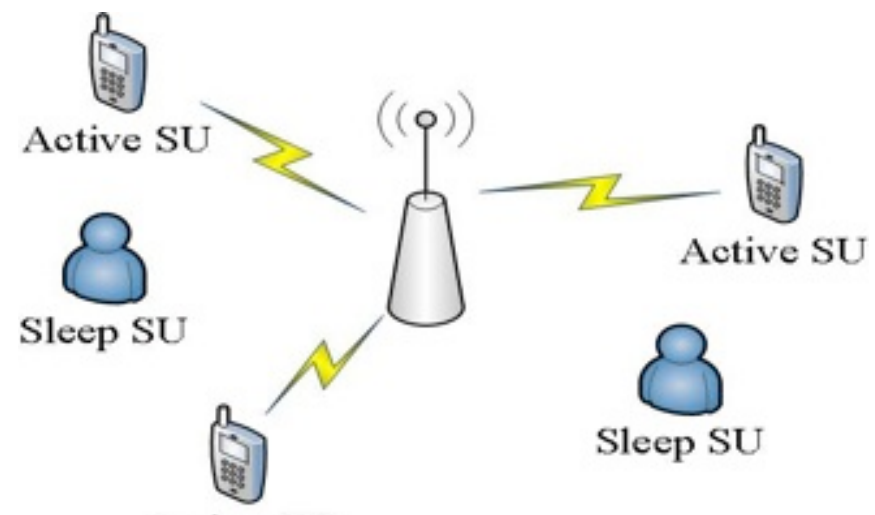

Active SU

Fig. (6). Network state diagram.

If it is considered that the conditions mentioned above are harsh, the requirements can be made milder and its feasibility can be proven using experiments. Now, it is not necessary for some SUs to send their sensing results when an $\mathrm{SU}$ has communication demand. FC just needs to fuse the sensing results in a period before the present moment. In order to simplify the problem, it can still be assumed that channels change uniformly and the number of SUs is in line with "Poisson Process". When the parameters of SUs are fixed, test results affected by the channels' changed cycle and FC' $s$ fusion period are shown in Fig. (7). As shown, the detection results perform best when the fusion period in $\mathrm{FC}$ is shorter than the changing cycle of channels, if there are enough sensing results.
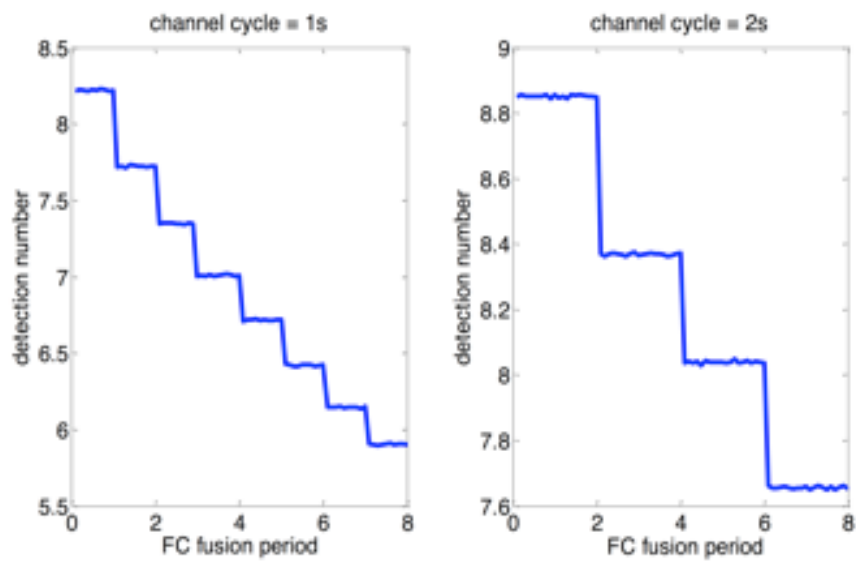

Fig. (7). Detection results affected by channel cycle. 
Now with the channel change cycle fixed for $1 \mathrm{~s}$, and the " $t$ " adjusted, a parameter of "Poisson Process", influences the number of SUs' sensing results in a period of time. Fig. (8) is obtained and a conclusion is drawn that with the sensing results reduced, the optimal fusion period is not always equal to channel change cycle.
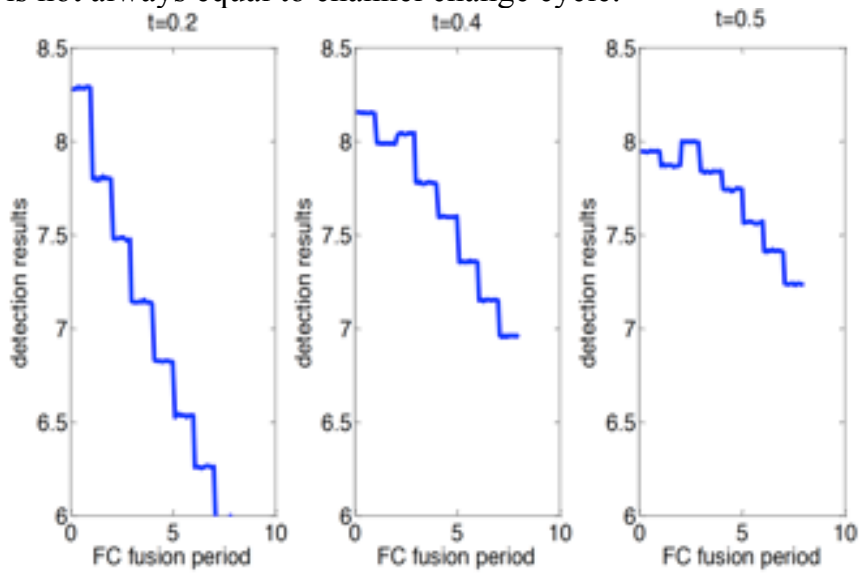

Fig. (8). Detection results affected by parameter "t".

Since the parameter " $t$ " can influence the last detection result, the average number of SUs influenced directly by it should be researched. Another parameter $\lambda=0.2$ is set in order to make the number change significantly. Fig. (9) shows that when the parameter " $\mathrm{t}$ " is greater than 0.4 , there will be no more than two SUs' sensing results sent to FC to finish data fusion. Thus, it cannot meet the basic requirements of cooperative perception.

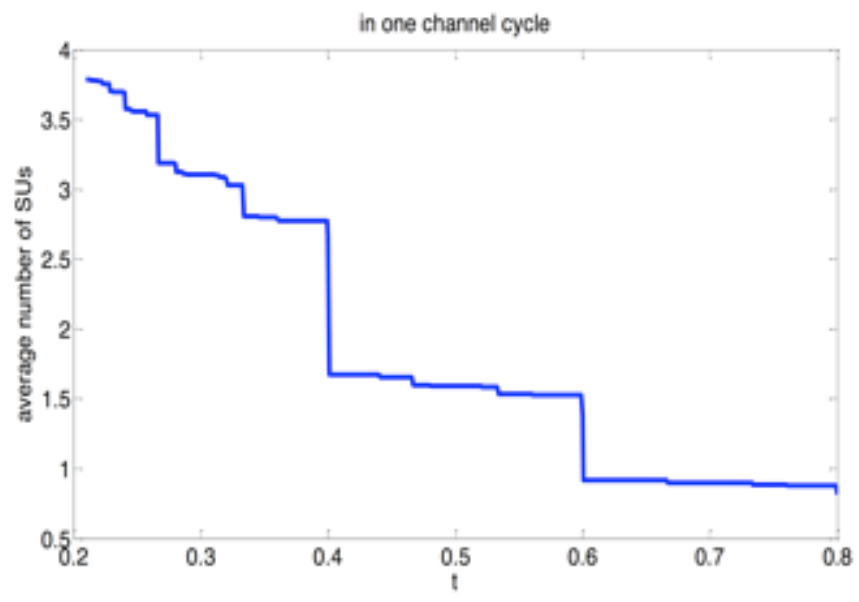

Fig. (9). Average number of SUs affected by parameter " $t$ ".

Now the conclusion can be drawn. If channels' average change cycle is known according to the statistics law and the average number of SUs is larger than 2.5 which send their sensing results to FC in one channel change cycle, the proposed scheme is applicable.

Since the feasibility of the proposed scheme is known, assuming that the number of $\mathrm{SU}$ is $\mathrm{N}\left(N \gg>N_{p p}\right)$ in the cognitive radio network and each SU's energy consumption for spectrum sensing is E. In a unit of time, there are $\mathrm{Na}$ active SUs and Ns sleep SUs $\left(N=N_{a}+N_{s}\right)$. So, the energy consumption of the proposed scheme is $(E \times N)$, compared to $(E \times N)$ of the traditional scheme. It can save a lot of energy especially in a large network.

This scheme can also ensure the fairness of the communication when saving energy. Some SUs may find their mobile terminals' batteries dead quickly because the traditional scheme asks all SUs to sense spectrum whether they need to communicate or not. It can also hinder the development of cognitive radio technology. The proposed scheme inherits the advantages of the technology of passive spectrum sensing and makes the cognitive radio technology more realistic.

\section{CONCLUSION}

The paper studied and optimized the collaborative spectrum sensing scheme for cognitive radio networks, including the perception time and the energy consumption. A passive spectrum sensing mode combined with perception service compensation was proposed in a centralized collaboration network structure. With the help of the advantages of centralized network and the flexible working of collaborative spectrum sensing, spectrum sensing time was shortened along with saving energy consumption. Theoretical analysis and simulation show that the proposed scheme not only meets the requirements of collaborative spectrum sensing in performance, but also saves energy. It is significant for mobile wireless communication which aspires to flexible mobile and lasting work.

\section{CONFLICT OF INTEREST}

The authors confirm that this article content has no conflict of interest.

\section{ACKNOWLEDGEMENTS}

The work presented in this paper was supported by the National Natural Science Foundation of China (Grants No. NSFC-61170176), Fund for the Doctoral Program of Higher Education of China (Grants No.20120005110002), National Great Science Specific Project (Grants No. 2012ZX 03005008,2012ZX03003006) and Beijing Municipal Commission of Education Build Together Project.

\section{REFERENCES}

[1] J. Mitola Cognitive radio, "An integrated agent architecture for software defined radio", Ph.D. Thesis, KTH Royal Institute of Technology, Sweden, 2000.

[2] Y. Liang, L. Lai, and J. Halloran, "Distributed algorithm for collaborative detection in cognitive radio networks", In: $47^{\text {th }}$ Annual Allerton Conference on Communication Control and Computing, 2009, pp. 394-399.

[3] H. Li, H. Dai, and C. Li, "Collaborative quickest spectrum sensing via random broadcast in cognitive radio systems", IEEE Global Telecommunications Conference, 2009, pp. 1-6.

[4] H. Teng-Cheng, W. Tsang-Yi, and H. Y.-W. Peter, "Collaborative change detection for efficient spectrum sensing in cognitive radio networks", In: IEEE Vehicular Technology Conference, 2010, pp. $1-5$.

[5] H. Li, M. Junfei, X. Fangmin, L. ShuRong, and Z. Zheng "Optimization of collaborative spectrum sensing for cognitive radio", In: Networking Sensing and Control, 2008, pp. 1730-1733.

[6] G. Amir, and S. Sousa Elvino, "Optimization of spectrum sensing for opportunistic spectrum access in cognitive radio networks," In IEEE Consumer Communications and Networking Conference, 2007, pp. $1022-1026$. 
[7] N. Armi, N.M. Saad, Y M. Zuki, and M. Arshad, "Cooperative spectrum sensing and signal detection in cognitive radio," In: IEEE International Conference on Intelligent and Advanced Systems, 2010, pp. 1-5.

[8] K. Arshad, and K. Moessner, "Collaborative Spectrum Sensing: Optimising the number of collaborating users," In: International Workshop on Cognitive Wireless Systems, 2009, pp. 1-5.

[9] H. Kim, and K.G. Shin, "Efficient discovery of spectrum opportunities with MAC-layer sensing in cognitive radio networks,"
IEEE Transactions on Mobile Computing, vol.7, pp. 533-545, 2008.

[10] G. Chen, P. Tao and W. Wenbo, "The optimization for the cooperative spectrum sensing mechanism in cognitive radio networks", Electronic \& Information Technology (China), vol. 31, pp. 1525-1530, 2009.

[11] Federal Communication Commission, "Spectrum Policy Task Force," Report Of the Spectrum Efficiency Working Group, 2002.

Received: November 11, 2013

Revised: February 11, 2014

Accepted: March 3, 2014

(C) Wen and Mu; Licensee Bentham Open

This is an open access article licensed under the terms of the Creative Commons Attribution Non-Commercial License (http://creativecommons.org/licenses/ by-nc/4.0/) which permits unrestricted, non-commercial use, distribution and reproduction in any medium, provided the work is properly cited. 